\title{
Portion size normality and additional within-meal food intake: two crossover laboratory experiments
}

\author{
Ashleigh Haynes ${ }^{1 * \dagger}+$, Charlotte A. Hardman², Jason C. G. Halford ${ }^{2}$, Susan A. Jebb ${ }^{3}$ and Eric Robinson ${ }^{2}$ \\ ${ }^{1}$ Centre for Behavioural Research in Cancer, Cancer Council Victoria, Melbourne, VIC 3004, Australia \\ ${ }^{2}$ Department of Psychological Sciences, University of Liverpool, Liverpool L69 7ZA, UK \\ ${ }^{3}$ Nuffield Department of Primary Care Health Sciences, University of Oxford, Oxford OX2 6GG, UK \\ (Submitted 17 January 2019 - Final revision received 28 August 2019 - Accepted 29 August 2019; electronically published 6 September 2019)
}

\section{Abstract}

Reducing food portion size could reduce energy intake. However, it is unclear at what point consumers respond to reductions by increasing intake of other foods. We predicted that a change in served portion size would only result in significant additional eating within the same meal if the resulting portion size was no longer visually perceived as 'normal'. Participants in two crossover experiments (Study 1: $n$ 45; Study 2: $n$ 37; adults, $51 \%$ female) were served different-sized lunchtime portions on three occasions that were perceived by a previous sample of participants as 'large-normal', 'small-normal' and 'smaller than normal', respectively. Participants were able to serve themselves additional helpings of the same food (Study 1) or dessert items (Study 2). In Study 1 there was a small but significant increase in additional intake when participants were served the 'smaller than normal' compared with the 'small-normal' portion ( $m$ difference $=161 \mathrm{~kJ}, P=0 \cdot 002, d=0 \cdot 35$ ), but there was no significant difference between the 'small-normal' and 'large-normal' conditions ( $m$ difference $=88 \mathrm{~kJ}, P=0 \cdot 08, d=0 \cdot 24$ ). A similar pattern was observed in Study $2(m$ difference $=149 \mathrm{~kJ}, P=0 \cdot 06, d=0 \cdot 18 ; m$ difference $=83 \mathrm{~kJ}, P=0 \cdot 26, d=0 \cdot 10)$. However, smaller portion sizes were each associated with a significant reduction in total meal intake. The findings provide preliminary evidence that reductions that result in portions appearing 'normal' in size may limit additional eating, but confirmatory research is needed.

Key words: Portion size: Food environment: Norms: Food portions: Obesity

Portion sizes of some common food products have increased over the past 40 years $^{(1-3)}$. Although a direct causal effect of increased portion sizes on population-level obesity has yet to be demonstrated $^{(4-7)}$, there is now plausible evidence that larger portion sizes promote increased food intake ${ }^{(8-11)}$. This has led to the suggestion that reductions to portion sizes of commercially available food products may reduce total energy intake and obesity $^{(2,4,12,13)}$. A small number of studies provide evidence suggesting that reducing the size of food portions can decrease food intake ${ }^{(14-16)}$; however, there is likely to be a point at which decreasing portion size will invite additional eating ${ }^{(15)}$, whereby consumers offset the reduction by consuming more of other foods. This may result in little or no overall benefit of reducing portion size, but it is currently unclear what determines the point at which reducing portion size prompts additional eating and ceases to reduce overall energy intake.

We previously proposed a theoretical model based on the social norms that may explain the influence of portion size on eating behaviour ${ }^{(17-22)}$ and that may be used to predict when additional eating in response to a reduced food portion size is likely to occur ${ }^{(23)}$. The 'norm range' model proposes that whether significant additional eating occurs is driven in part by the visual perception of whether a portion is categorised as being 'normal' in size, and not solely by its objective size or energy content. While the perceived normality of portion sizes may be malleable, we speculate that the range of portions that are perceived as 'normal' by an individual will be largely stable. We also speculate that there will be similarity between individuals, as previous work has demonstrated considerable overlap between the range of portion sizes perceived as 'normal' by independent samples of participants ${ }^{(23)}$. If a portion is perceived to be 'normal' in size, a consumer is likely to intend to and subsequently consume most of a portion without requiring additional food. However, a portion that is perceived as 'smaller than normal' is likely to invite intake of additional food, whereby a consumer may attempt to offset the perceived deficit by subsequently consuming more of other food. Because there are a range of portions that are perceived as 'normal', any reductions

* Corresponding author: Ashleigh Haynes, email ashleigh.haynes@cancervic.org.au

$\dagger$ This work was carried out while the first author was employed at the University of Liverpool. 


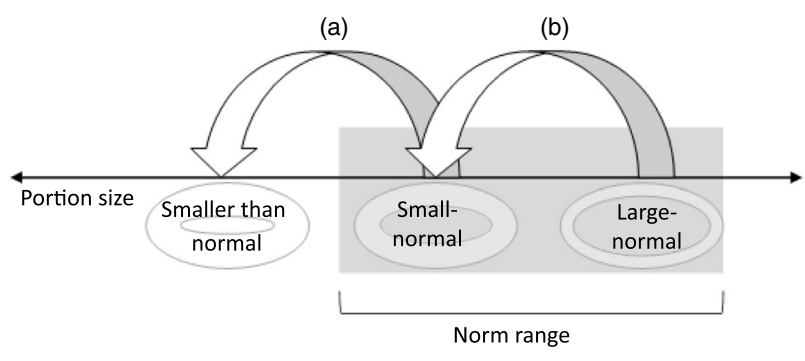

Fig. 1. Norm range model. Norm range = range of portions visually perceived as 'normal' by an independent sample of participants. (a) and (b) represent equivalent changes to portion size. Significantly greater additional intake hypothesised for (a) comparison of 'smaller than normal' to 'small-normal', but not (b) comparison of 'small-normal' to 'large-normal'. Significant reduction in overall energy intake hypothesised for (b) comparison of 'small-normal' to 'large-normal' but not (a) comparison of 'smaller than normal' to 'small normal'.

to portion size that occur within this range are likely to reduce intake. However, a reduction that results in a portion being perceived as 'smaller than normal' is likely to result in additional eating and therefore may not reduce overall intake. Accordingly, rather than consumers achieving an equivalent level of energy intake regardless of the size of the initial portion, the amount of additional intake may be biased by whether the initial portion is visually categorised as 'normal' in size. These predictions were supported in two virtual experiments which assessed ratings of intended consumption of an initial portion and of additional food $^{(23)}$. However, the role of perceived normality of portion size in influencing whether humans engage in actual additional eating in response to a reduced portion size is yet to be examined.

In the present research, we tested whether the norm range model could be used to predict when a change to the portion size of a main component of a lunchtime meal would exert a substantial influence on additional food intake, over and above the initial portion, leading to an increase in energy intake within a single meal. Across two studies, we measured intake from a lunchtime meal that featured a main component in one of three portion sizes in a counterbalanced order: two portions that were perceived as 'normal' by an independent sample of participants (one 'large-normal' and one 'small-normal'), and a 'smaller than normal' portion (Fig. 1). In addition to the initial portion of the main meal component, additional food was made available from which participants could serve themselves if desired (which represented additional intake). In Study 1 , we examined additional intake of more of the same food (resembling a single-course meal structure with optional 'additional helpings' of the same food). Sensory-specific satiation, the phenomenon whereby appetite for a consumed food decreases relative to a food that is not consumed ${ }^{(24)}$, may limit the extent of additional eating of the same food after a reduced portion. Therefore, Study 2 examined additional intake of self-served 'dessert' food (from a selection of two different types) after participants' initial portion of a main course, to resemble a two-course meal structure.

We expected that participants would consume all or most of the portions initially served, and in line with our proposed norm range theory, we hypothesised that consumers would engage in greater additional eating beyond the initial served portion after consuming a 'smaller than normal' portion than a 'small-normal' portion (Fig. 1(a)), but that additional intake would not significantly differ between the two 'normal' portions (Fig. 1(b)), despite each comparison representing the same difference in food weight. We also predicted that total meal energy intake in the 'smaller than normal' portion would not be lower than in the 'small-normal' portion size condition because of the extent of additional eating induced by 'smaller than normal' portions.

\section{Methods}

\section{Participants}

The studies were advertised to university staff and students and in the local community as investigating 'appetite and word categorisation' (Study 1) and 'mood stability' (Study 2) to blind participants to the study aims. Individuals with food allergies, intolerances or specific dietary requirements (including being vegetarian or vegan) or a history of eating disorders were ineligible to participate; and participants were screened for general liking and willingness to consume the test foods in each study. We recruited adults with a self-reported BMI between 22.5 and $32.5 \mathrm{~kg} / \mathrm{m}^{2}$, because the BMI of approximately $70 \%$ of adults in England fall within this range ${ }^{(25)}$. We aimed to recruit an equal number of males and females and an equal number of participants in two BMI bands $\left(22 \cdot 5-27.49\right.$ and $\left.27 \cdot 5-32.5 \mathrm{~kg} / \mathrm{m}^{2}\right)$ to avoid overrepresentation of participants with lower BMI values. Eligibility (including BMI based on self-reported height and weight) was assessed using an online questionnaire.

\section{Study foods}

The served lunchtime portion sizes of the main meal component in each study were selected based on portions reliably identified as 'normal' and 'not normal' in size by the majority of an independent sample of participants recruited from the same population (university staff and students and in the local community) using the same recruitment strata in a recent study ${ }^{(23)}$. In this previous study, participants viewed images of each meal varying in portion size and judged whether each portion was 'normal' or 'not normal' in size. The 'norm range' (the range perceived as 'normal' by $\geq 60 \%$ of the sample) encompassed portion sizes from 70 to $120 \%$ of the manufacturer's recommended serving of pasta with tomato sauce, and $80-160 \%$ of the manufacturer's recommended serving of chicken curry with rice ${ }^{(23)}$.

In Study 1, participants were served an initial portion of pasta (Tesco Everyday Value quick cook penne, $640 \mathrm{~kJ} / 100 \mathrm{~g}$ ) with tomato sauce (Tesco Everyday Value pasta sauce, $138 \mathrm{~kJ} / 100 \mathrm{~g}$ ). A fixed ratio of pasta and sauce was prepared and mixed according to standardised instructions, and then was served in the appropriate portion size on a standard-sized white dinner plate $(255 \mathrm{~mm}$ diameter). In the 'large-normal' condition, participants were served a portion that was equal to $120 \%$ of the manufacturer's recommended serving size $(336 \mathrm{~g}, 1284 \mathrm{~kJ})$. The portion was reduced to $90 \%$ of the recommended serving in the 'small-normal' condition ( $252 \mathrm{~g}, 962 \mathrm{~kJ}$ ), and to $60 \%$ in the 'smaller than normal' condition (168 g, $644 \mathrm{~kJ}$ ). A serving bowl containing an additional $200 \%$ of the recommended serving size of pasta and tomato sauce was placed on a hot plate located on a serving counter located behind the participant at the same time as the initial portion was served, to allow them to refill their plate if desired. 
In Study 2, participants were served an initial portion of chicken curry (Tesco Chicken Curry, $377 \mathrm{~kJ} / 100 \mathrm{~g}$ ) with rice (Tesco Microwave Long Grain Rice, 699 kJ/100 g). The portions of curry and rice were individually weighed before being cooked according to a standardised procedure. The curry and rice were served in a standardised manner on a standard white dinner plate, such that the curry and rice were touching but not mixed. Participants were served a portion that was equal to $130 \%$ of the manufacturer's recommended serving size ( $423 \mathrm{~g}, 2117 \mathrm{~kJ})$ in the 'large-normal' condition, $100 \%$ in the 'small-normal' condition ( $325 \mathrm{~g}, 1628 \mathrm{~kJ})$ and $70 \%$ in the 'smaller than normal' condition (228 g, $1138 \mathrm{~kJ}$ ). Participants were provided with a dessert buffet of two bowls containing bite-sized pieces of caramel shortbread (Tesco Millionaire Bites, $2092 \mathrm{~kJ} / 100 \mathrm{~g}$, ten pieces, approximately $120 \mathrm{~g}, 2406 \mathrm{~kJ}$ ), and flapjack (Tesco Mini Flapjack Bites, $1916 \mathrm{~kJ} / 100 \mathrm{~g}$, ten pieces, approximately $150 \mathrm{~g}, 2874 \mathrm{~kJ}$ ), respectively. The dessert buffet was located behind the participant at the same time as the initial portion was served, to allow them to serve themselves dessert if desired. See online Supplementary materials for macronutrient content of all meal components.

\section{Measures}

Hunger and fullness. To assess pre- and post-meal hunger and fullness, participants indicated their current level of hunger and fullness on a 100-mm computerised visual analogue scale ranging from 0 ('not at all') to 100 ('extremely'). Hunger and fullness ratings were presented in a series of mood ratings (e.g. 'how calm are you right now?').

Perceived portion size normality. As a manipulation check, participants were shown an image of each portion size served during the study in a randomised order and were asked: 'In your opinion, how normal is the portion of pasta/chicken curry and rice shown below? By "normal" we mean whether the portion contains a normal amount of food to eat for a single meal.' Responses were provided on seven-point Likert scales ranging from 1 ('not normal, it is too small') to 7 ('not normal, it is too big'), with a midpoint of 4 ('normal').

Usual portion. In Study 1, participants viewed images of pasta with tomato sauce portions (ranging from 50 to $200 \%$ of the manufacturer's recommended serving size at $10 \%$ increment increases in portion size). The images were presented simultaneously and participants were asked to indicate which portion was closest to the amount of pasta with tomato sauce they would usually serve themselves.

In Study 2, participants completed a computer-based task programmed in Psychopy to indicate the portion size of chicken curry and rice closest to their usual serving size. The task began with the presentation of an image displaying a portion size of chicken curry with rice equal to $40 \%$ of the manufacturer's recommended serving size. Participants adjusted the size of the displayed portion using the up and down arrow keys until it appeared equivalent to the amount of that food they would usually serve themselves, when they pressed 'enter' to select the portion size. Each arrow key press increased or decreased the portion by an increment of $10 \%$ of the recommended serving, to a maximum of $300 \%$.

\section{Procedure}

Participants took part in either Study 1 or Study 2 and completed one lunchtime testing session per condition, separated by a washout period of between 7 and $10 \mathrm{~d}$. Each condition of the design was completed in a counterbalanced (Study 1) or randomised order (Study 2, using 'RANDBETWEEN' function in Microsoft Excel to assign participant to one of six sequences). Participants were asked not to consume any energy-containing food or drink for $2 \mathrm{~h}$ preceding each session (scheduled to commence between 12.00 and 14.00 hours). During each session, participants first reported how long since they had last eaten, and in Study 1, they completed a short computer-based filler task ostensibly measuring word categorisation speed (to bolster the cover story). Participants then completed pre-meal hunger and fullness ratings before sitting at an empty table, and a researcher then served the lunch by placing the initial portion (pasta, Study 1; curry with rice, Study 2) in front of them. The researcher informed participants that once they had finished their served portion they could refill their plate with more pasta from the serving bowl (Study 1) or serve themselves from the dessert buffet using tongs onto a small side plate (Study 2) at their discretion, and then left the participant to consume the meal alone. Participants were provided with as much time as they needed to finish eating and were not required to finish the initial portion served. Participants then completed post-meal hunger and fullness ratings and a post-meal word categorisation task (identical to pre-meal task) in Study 1 or moodrelated filler measures in Study 2 (see online Supplementary materials). The weight of food consumed was calculated by measuring the amount served and amount leftover using digital scales (Sartorius). Food weights were recorded in grams to the nearest $0.1 \mathrm{~g}$ in Study 1 , and to the nearest $0.01 \mathrm{~g}$ in Study 2, due to a change in measurement equipment. Separate weights were taken for each distinct meal component (e.g. plated pasta portion, 'extra' pasta portion, rice, curry, and each dessert component). At the end of the final session, participants reported what they thought was the aim of the study (free text response), completed the remaining on-screen self-report measures (including perceived portion size normality for each condition and a standard battery of measures assessing eating habits and preferences; see online Supplementary materials), and a researcher measured their height using a stadiometer (Seca) and weight using a digital scale (Salter), before debriefing. In Study 1, height was recorded to the nearest $0 \cdot 1 \mathrm{~cm}$, and weight to the nearest $0 \cdot 1$ $\mathrm{kg}$. In Study 2, height was recorded to the nearest 0.5 or $0.1 \mathrm{~cm}$ and weight to the nearest $0 \cdot 1$ or $0.05 \mathrm{~kg}$. Participants completed each testing session individually in a quiet room. Study 1 was conducted between October and December 2016, and Study 2 was conducted between May and July 2017. Both studies were conducted in line with institutional ethical approval (IPHS1516-LB-252-Generic RETH000955, IPHS-1617-LB-277-Generic RETH000955), and participants provided informed consent at the beginning of their first session and were provided with a 
financial incentive to participate (£30). Study 2 was pre-registered on the Open Science Framework (https://osf.io/txf9u/), and the research is registered on ClinicalTrials.gov (NCT03731273)

\section{Analysis plan}

Power calculation. For Study 1, we calculated that a minimum sample size of thirty-four would be sufficient to detect a mediumsized effect of portion size on energy intake with $80 \%$ power and an $\alpha$-level of 0.05 using a repeated-measures ANOVA $\left(f^{2}=0.25\right.$, correlation between repeated measures $=0.5$, non-sphericity correction $=0.75, G^{*}$ Power 3.1$)^{(9)}$. We calculated that Study 2 required a sample size of thirty-five to be adequately powered (80\%) to detect a difference between additional intake after the initial portion in the 'smaller than normal' and 'small-normal' conditions that was observed in Study 1 (group parameters for power calculation: 'smaller than normal': $m=519(\mathrm{sD} 479) \mathrm{kJ}$; 'small-normal': $m=358$ (sD 432) kJ; correlation between conditions, $r 0 \cdot 74)$. We aimed to recruit forty-eight participants in Study 1 and forty participants in Study 2 to allow for potential exclusions and to ensure an equal representation of participants in each sex and BMI band.

Primary analyses. All analyses were conducted in IBM SPSS 24.0 and were pre-registered for Study 2 unless otherwise stated. Effect sizes (Cohen's $d$ ) can be interpreted as small $(0 \cdot 2)$, medium $(0 \cdot 5)$ and large $(0 \cdot 8)^{(26)}$. Three repeated-measures ANOVA (with pairwise comparisons to interpret significant main effects) were conducted to examine the effect of portion size (smaller than normal, small-normal, large-normal) on (a) energy intake from the initial served portion, (b) additional energy intake (amount consumed from the extra bowl of lunch food or dessert buffet), and (c) total energy intake (sum of (a) and (b)). Where non-sphericity was detected by a significant Mauchly's test, Greenhouse Geissercorrected ANOVA results are reported (indicated by adjusted df to two decimal places)

Sensitivity analyses. Two researchers independently coded the open-ended responses to the aim guessing question as 'aware' of study aims if the participant referred to the influence of portion or serving size of food on how much was eaten. Two sets of sensitivity analyses were conducted. The analysis of the effect of portion size on energy intake was repeated excluding (a) participants who guessed the aims of the study, and then (b) participants with outlying total or additional energy intake in any portion size condition ( $>2.5$ SD from condition mean). We report whether these exclusions result in deviations from the pattern of significance of the main analyses (i.e. any significant differences between conditions becoming NS, and vice versa).

Secondary analyses. To compare changes in hunger and fullness from pre- to post-meal between portion size conditions, we conducted two 3 (portion size) $\times 2$ (time: pre-, post-meal) repeated-measures ANOVA. Significant interactions were followed up by examining differences in baseline hunger and fullness between conditions, and significant baseline differences were followed up with sensitivity analyses testing the main hypotheses using linear mixed models controlling for baseline appetite ratings.

Perceived normality of the served portion sizes was compared between portion size conditions using a repeated-measures ANOVA, and one-sample $t$ tests were conducted to investigate whether participants perceived the portions to be significantly different from normal by comparing the mean perceived normality with the midpoint of the scale. In a post boc exploratory analysis suggested by an anonymous reviewer, we also examined the correlation between the perceived normality rating of each portion size and additional intake in the respective portion size condition, and calculated a single aggregated correlation between perceived normality and additional intake across conditions within each study (using R package 'rmcorr' for repeated-measures correla$\left.\operatorname{tion}^{(27)}\right)$. We also report the median self-reported 'usual portion size' of each of the served main meal foods for comparison with the presented portion sizes.

To explore order effects, we tested whether the sequence in which participants were served the three portions moderated the effect of portion size on energy intake by testing the interaction between portion size condition and a between-subjects variable representing portion size sequence using a 6 (representing condition sequence) $\times 3$ mixed ANOVA for each energy intake variable. This analysis was not pre-registered but was conducted to test the robustness of the portion size effects.

\section{Results}

\section{Sample characteristics}

We recruited forty-nine participants for Study 1. One participant withdrew after session 1 due to a scheduling conflict; two participants were served the same portion size in two sessions in error; and one participant's BMI was $>2.5$ sD from the sample mean $\left(\mathrm{BMI}=42 \cdot 2 \mathrm{~kg} / \mathrm{m}^{2}\right)$ and was excluded from the analysis as decided a priori. Conducting the analyses with and without this participant yielded the same pattern of results (i.e. none of the statistically significant findings become non-significant, and vice versa). The final sample ( $n$ 45; twenty-three women) had a mean BMI of 26.9 (sD 3.7; range 18.9-35.3) kg/m²; BMI < 27.5 kg/m², $n$ 24; BMI $\geq 27.5 \mathrm{~kg} / \mathrm{m}^{2}, n$ 21) and a mean age of 30.4 (SD $12 \cdot 7$; range 18-76) years. Participant eligibility was assessed using selfreported height and weight, while the reported sample characteristics are based on researcher-measured height and weight in the final session. The measured BMI of the sample exceeds the recruitment cut-off points because of errors in participant selfreport. Seven participants were aware of the aim of the study, and there were three outliers on either total or additional intake in at least one condition. As decided a priori, data from these participants are included in the reported analyses, but the significance of the results did not vary depending on their inclusion.

For Study 2, forty-one participants were recruited, as two participants withdrew after the first session due to a scheduling conflict. An additional two participants were served the same portion size in two sessions in error, leaving a final sample of thirty-seven (nineteen women) with a mean BMI of 26.9 (SD 3.7, range 20.1$35.5) \mathrm{kg} / \mathrm{m}^{2}$; BMI $<27.5 \mathrm{~kg} / \mathrm{m}^{2}, n 20$; BMI $\left.\geq 27.5 \mathrm{~kg} / \mathrm{m}^{2}, n 17\right)$ and a mean age of 32.2 (SD $12 \cdot 2$; range 20-59) years. Seven 

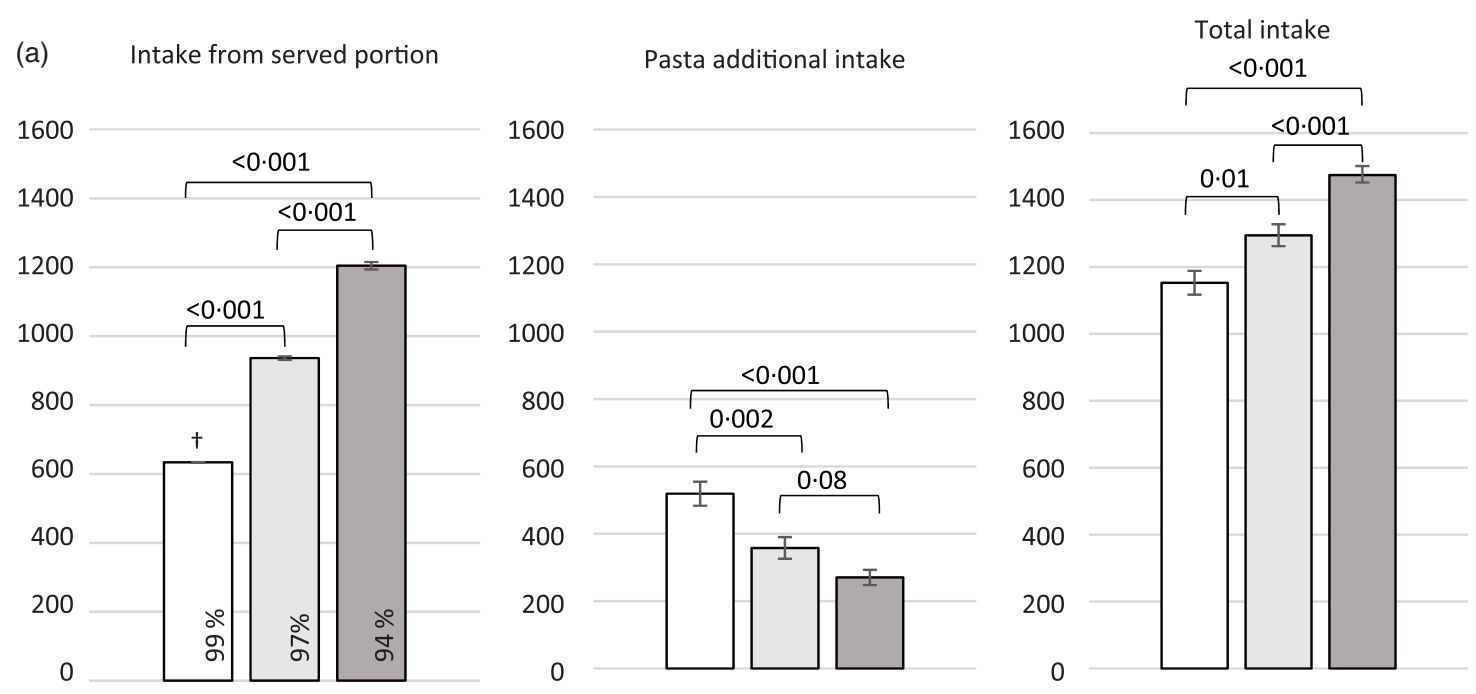

(b) Intake from served portion

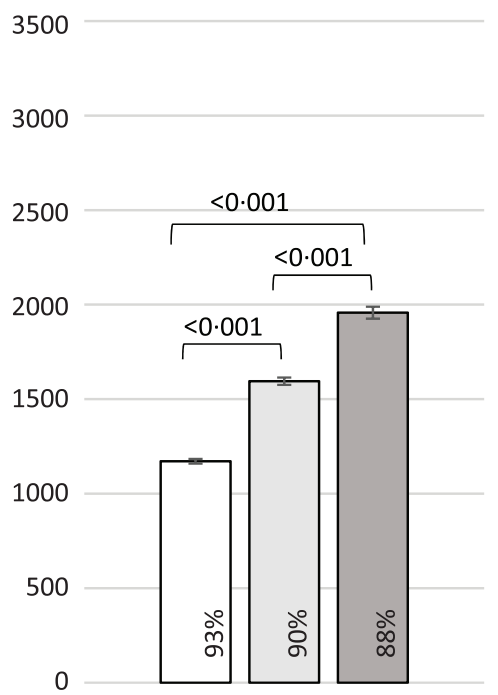

3500

3000

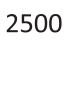

2000

1500
1000
500
0
Dessert additional intake

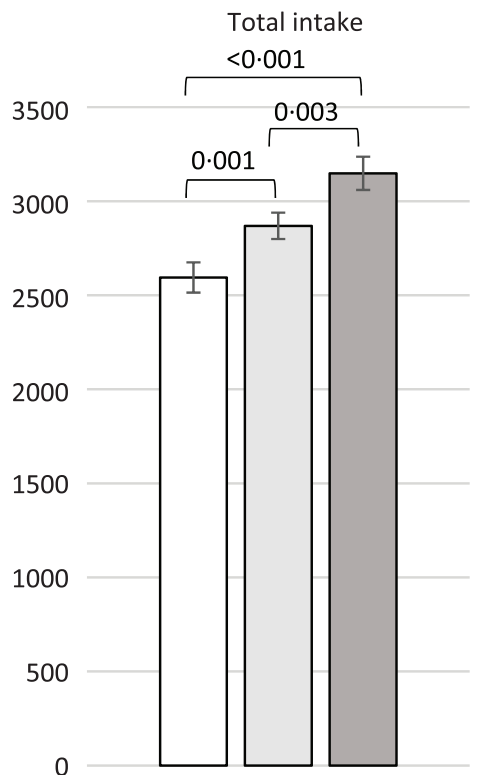

Fig. 2. Energy intake (kJ) by portion size condition for Study 1 (a) and Study 2 (b). Percentage values represent intake as a percentage of served portion. Error bars represent standard errors. Values on comparison bars are $P$ for pairwise comparisons. ${ }^{*} P<0.05$ in pre-registered sensitivity analyses. ( $\square$ ), Smaller than normal; $(\square)$, small-normal; $(\square)$, large-normal. † SE $=1.08$.

participants were aware of the aims of the study, and one participant's total and additional energy intake in the small-normal condition was $>2.5$ sD from the condition mean. As decided a priori, analyses are reported including data from participants who guessed the aims and outliers on energy intake. Except where indicated in footnotes, the significance of the results did not vary depending on whether these participants were included or excluded. See online Supplementary Fig. S1 for a Consolidated Standards of Reporting Trials (CONSORT) flow diagram.

\section{Effect of portion size on intake from initial portion}

Fig. 2 displays energy intake from the initial portion (and mean percentage of the initial portion consumed), energy intake from additional self-served food, and total energy intake across portion size conditions in both studies. In Study 1, there was a significant effect of portion size on energy intake from the initial portion, $F_{1.34}, 59.06=530.54, P<0.001$, partial $\eta^{2}=0.92$ (Fig. 2(a)). As predicted, participants consumed significantly less from the 'smaller than normal' portion than the 'small-normal' portion ( $m$ difference $=302.5 \mathrm{~kJ}, \mathrm{se}=10 \cdot 3, P<0 \cdot 001, d=6 \cdot 28$ ), and the 'large-normal' portion ( $m$ difference $=570 \cdot 6 \mathrm{~kJ}$, $\mathrm{SE}=21.7, P<0.001, d=5.56)$; and ate significantly less from the 'small-normal' portion than from the 'large-normal' portion ( $m$ difference $=268.1 \mathrm{~kJ}, \mathrm{sE}=18.5, P<0.001, d=2.37$ ). Likewise, in Study 2 there was a significant effect of portion size on energy intake from the initial served portion, $F_{1 \cdot 22,43.96}=194 \cdot 80, P<0 \cdot 001$, partial $\eta^{2}=0.84$ (Fig. 2(b)). Participants consumed significantly less from the 'smaller than normal' portion than the 'smallnormal' portion ( $m$ difference $=423.4 \mathrm{~kJ}, \quad \mathrm{sE}=29.5, \quad P<0.001$, $d=2 \cdot 16$ ), and the 'large-normal' portion ( $m$ difference $=786 \cdot 0 \mathrm{~kJ}$, $\mathrm{SE}=53.4, \quad P<0.001, d=2.69)$; and ate significantly less from 
Table 1. Pre- and post-meal hunger and fullness by portion size condition (Mean values and standard deviations)

\begin{tabular}{|c|c|c|c|c|c|c|c|c|c|c|c|c|c|}
\hline & & \multicolumn{6}{|c|}{ Study 1} & \multicolumn{6}{|c|}{ Study 2} \\
\hline & & \multicolumn{2}{|c|}{$\begin{array}{c}\text { Smaller than } \\
\text { normal }\end{array}$} & \multicolumn{2}{|c|}{ Small-normal } & \multicolumn{2}{|c|}{ Large-normal } & \multicolumn{2}{|c|}{$\begin{array}{c}\text { Smaller than } \\
\text { normal }\end{array}$} & \multicolumn{2}{|c|}{ Small-normal } & \multicolumn{2}{|c|}{ Large-normal } \\
\hline & & Mean & SD & Mean & SD & Mean & SD & Mean & SD & Mean & SD & Mean & SD \\
\hline \multirow[t]{2}{*}{ Pre-meal } & Hunger & $59 \cdot 40^{a, b}$ & $23 \cdot 18$ & $67 \cdot 07^{a}$ & $22 \cdot 34$ & $70 \cdot 16^{b}$ & 20.83 & $62 \cdot 35$ & $22 \cdot 18$ & 64.73 & 23.03 & $66 \cdot 35$ & $22 \cdot 10$ \\
\hline & Fullness & $21 \cdot 44^{\mathrm{a}}$ & 19.63 & 18.44 & $20 \cdot 38$ & $14.56^{a}$ & 20.56 & $14 \cdot 14$ & $16 \cdot 01$ & 13.97 & $14 \cdot 74$ & 14.65 & 14.85 \\
\hline \multirow[t]{2}{*}{ Post-meal } & Hunger & 8.09 & $15 \cdot 81$ & 6.33 & 8.54 & 7.89 & 12.85 & 4.27 & $5 \cdot 15$ & 9.22 & 11.75 & 8.78 & 9.48 \\
\hline & Fullness & 83.36 & $15 \cdot 91$ & 86.91 & 11.60 & $85 \cdot 16$ & $15 \cdot 39$ & 85.65 & 11.93 & 77.38 & 13.06 & 77.32 & 19.92 \\
\hline
\end{tabular}

a,b Mean values for Study 1 with common superscripts on the same row significantly differ between conditions $(P<0.05)$. No pairwise comparisons were conducted for Study 2 as there was no main effect of condition or time $\times$ condition interaction.

the 'small-normal' portion than from the 'large-normal' portion ( $m$ difference $=362 \cdot 6 \mathrm{~kJ}, \mathrm{sE}=32 \cdot 3, P<0 \cdot 001, d=1 \cdot 14$ ).

\section{Effect of initial portion size on additional intake after the initial portion}

In Study 1, there was a significant effect of portion size on additional intake of pasta, $F_{2,88}=12 \cdot 70, P<0 \cdot 001$, partial $\eta^{2}=0 \cdot 22$ (Fig. 2(a)). Consistent with predictions, additional intake did not significantly differ between the 'small-normal' and 'large-normal' conditions ( $m$ difference $=87.6 \mathrm{~kJ}, \mathrm{sE}=49 \cdot 2, P=0.08, d=0.24)$, but was significantly higher in the 'smaller than normal' than in the 'largenormal' condition ( $m$ difference $=248.6 \mathrm{~kJ}, \mathrm{sE}=51.9, P<0.001$, $d=0.62$ ), and in the 'smaller than normal' than the 'small-normal' condition ( $m$ difference $=161.0 \mathrm{~kJ}, \mathrm{sE}=49 \cdot 0, P=0.002, d=0.35$ ).

In Study 2, portion size condition had a significant effect on additional energy intake from the self-served dessert buffet, $F_{2,72}=4.66, P=0.01$, partial $\eta^{2}=0.12$ (Fig. 2(b)). Consistent with predictions, additional intake did not significantly differ between the 'small-normal' and 'large-normal' conditions ( $m$ difference $=$ $83.4 \mathrm{~kJ}, \mathrm{sE}=72 \cdot 7, P=0 \cdot 26, d=0 \cdot 10$ ). Additional intake was significantly higher in the 'smaller than normal' than in the 'largenormal' condition $(m$ difference $=232 \cdot 4 \mathrm{~kJ}, \mathrm{sE}=81 \cdot 7, P=0 \cdot 01$, $d=0.26$ ). Additional intake was also higher in the 'smaller than normal' than the 'small-normal' condition, but this difference was small in magnitude and not statistically significant $(m$ difference $=$ $148.9 \mathrm{~kJ}, \mathrm{SE}=76.7, P=0.06, d=0.18$ ). However, the difference in additional intake between the 'smaller than normal' and 'small-normal' conditions was statistically significant after excluding participants who were aware of the study aims and one outlier. Additional eating was significantly higher in the 'smaller than normal' portion condition than the 'small-normal' condition in Study 2 when participants who guessed the study aims were excluded from the analysis $(m$ difference $=166.0 \mathrm{~kJ}, \mathrm{sE}=76.7, P=0.04, d=0.21$ ), and when the participant with outlying additional energy intake was excluded $(m$ difference $=159.6 \mathrm{~kJ}, \mathrm{SE}=78.1, P=0.049$, $d=0 \cdot 19)$

\section{Effect of portion size on total meal energy intake}

In Study 1, total meal energy intake was significantly different between portion size conditions, $F_{2,88}=20.93, P<0.001$, partial $\eta^{2}=0.32$ (Fig. 2(a)). Participants ate significantly less overall in the 'small-normal' portion size condition than in the 'large-normal' condition $(m$ difference $=180 \cdot 6 \mathrm{~kJ}, \mathrm{sE}=51 \cdot 9, P=0.001, d=0.44)$, but contrary to predictions, participants also ate significantly less overall in the 'smaller than normal' portion size condition than in the 'small-normal' condition ( $m$ difference $=141.4 \mathrm{~kJ}, \mathrm{sE}=47 \cdot 8$, $P=0 \cdot 01, d=0.30$ ), indicating that additional eating in the 'smaller than normal' condition only partially offset the smaller size of the initial portion. Participants also ate significantly less in the 'smaller than normal' than in the 'large-normal' condition, $m$ difference $=$ $322 \cdot 0 \mathrm{~kJ}, \mathrm{SE}=49.8, P<0.001, d=0.76$

The same pattern of results was observed in Study 2. Portion size condition significantly affected total energy intake, $F_{2,72}=20 \cdot 57$, $P<0.001$, partial $\eta^{2}=0.36$ (Fig. 2(b)), and as in Study 1, participants ate significantly less overall in the 'small-normal' than in the 'largenormal' portion size condition ( $m$ difference $=279 \cdot 1 \mathrm{~kJ}, \mathrm{sE}=88 \cdot 1$, $P=0.003, d=0.29$ ), but also ate significantly less overall in the 'smaller than normal' portion size condition than in the 'smallnormal' condition ( $m$ difference $=274.5 \mathrm{~kJ}, \mathrm{SE}=77 \cdot 6, P=0.001$, $d=0.30$ ). Participants also ate significantly less in the 'smaller than normal' than in the 'large-normal' condition in Study 2, $m$ difference $=553.6 \mathrm{~kJ}, \mathrm{sE}=92.6, P<0.001, d=0.54$.

\section{Hunger and fullness}

In Study 1 there was a significant interaction between time (prepost) and portion size condition on hunger and fullness, explained by pre-meal appetite being lower in the 'smaller than normal' condition (Table 1). Separate linear mixed models testing the main hypotheses in Study 1 while controlling for pre-meal appetite revealed results consistent with the primary analyses, except that in line with our theoretical predictions, there was no significant difference in total meal intake between 'smaller than normal' and 'small normal' portion size conditions, and a marginally significant difference in total meal energy intake between 'large normal' and 'small normal' portions. There was no significant main effect of condition or interaction between condition and time predicting appetite ratings in Study 2 (see online Supplementary materials for full results and Table 1 for mean hunger and fullness ratings).

\section{Perceived normality of portion sizes and 'usual' portion size}

In Study 1, perceived normality significantly varied between portion sizes, $F_{2,88}=113.24, P<0.001$, partial $\eta^{2}=0.72$ (Table 2). Pairwise comparisons confirmed that perceived normality was significantly lower for the 'smaller than normal' than 'small-normal' 
Table 2. Perceived normality of portion sizes and correlation with additional intake†

(Mean values and standard deviations; correlations)

\begin{tabular}{|c|c|c|c|c|c|c|}
\hline & \multicolumn{2}{|c|}{$\begin{array}{c}\text { Smaller than } \\
\text { normal }\end{array}$} & \multicolumn{2}{|c|}{$\begin{array}{l}\text { Small- } \\
\text { normal }\end{array}$} & \multicolumn{2}{|c|}{$\begin{array}{l}\text { Large- } \\
\text { normal }\end{array}$} \\
\hline & Mean & SD & Mean & SD & Mean & SD \\
\hline $\begin{array}{l}\text { Study } 1 \\
\text { Correlation }\end{array}$ & $-0.54^{\star \star \star}$ & ${ }_{*}^{1.1}$ & \multicolumn{2}{|c|}{$-0.41^{\star *}$} & \multicolumn{2}{|c|}{-0.27} \\
\hline $\begin{array}{l}\text { Study } 2 \\
\quad \text { Correlation }\end{array}$ & ${ }^{3.5}-c$ & 1.0 & 4.6 & 0.8 & $\begin{array}{l}5 \cdot 6 \\
-0\end{array}$ & 1.0 \\
\hline
\end{tabular}

${ }^{*} P<0.05,{ }^{* *} P<0.01,{ }^{* * *} P<0.001$.

†'Smaller than normal' ( $v$. 'small-normal') and 'small-normal' ( $v$. 'large-normal') portion sizes were associated with significantly lower perceived normality ratings in both studies $(P<0.001)$. Perceived normality scale: 1 ('not normal, it is too small') to 7 ('not normal, it is too big') with a midpoint of 4 ('normal').

portion sizes, and for the 'small-normal' than 'large-normal portions. One-sample $t$ tests showed that the mean normality rating for the 'smaller than normal' portion size was significantly lower, $t(44)=-6.96, P<0.001, d=1.00$, and the normality rating for 'large-normal' portion size was significantly higher, $t(44)=6.09$, $P<0.001, d=0.91$, than the test value of 4 (corresponding to the midpoint of the scale, labelled 'normal'). The mean normality rating for the 'small-normal' portion size was not significantly different from the midpoint of the scale, $t(44)=0 \cdot 56, P=0.58, d=0.18$.

Likewise, perceived normality significantly varied between portion sizes in Study 2, $F_{1 \cdot 69,60.92}=79.34, P<0.001$, partial $\eta^{2}=0.69$. The mean normality rating for 'smaller than normal' was significantly lower, $t(36)=-2 \cdot 83, P=0 \cdot 01, d=0.50$, and for largenormal' was significantly higher, $t(36)=9.15, P<0.001, d=1.60$, than the midpoint of the scale, but so was the normality rating for the 'small-normal' portion size, $t(36)=4.76, P<0.001, d=0.75$.

Consistent with the norm range model, ratings on the perceived normality scale were negatively correlated with additional intake in each respective portion size condition with 'higher than normal' ratings predicting lower intake and 'lower than normal' ratings predicting lower intake, although this was not statistically significant across all conditions (Table 2). Similarly, the aggregated correlation between ratings of perceived normality and additional intake in each respective condition indicated that higher perceived normality ratings were associated with lower additional intake overall, but this correlation was significant in Study 1 $(r-0 \cdot 35, P<0 \cdot 001)$, but not in Study $2(r-0 \cdot 22, P=0.056)$.

Participants' self-reported usual portion size of the lunch food tended to fall between the 'small normal' and 'large normal' portions in Study 1 but was closer to the 'smaller than normal' portion size in Study 2. See online Supplementary materials.

\section{Order effects}

The order in which participants completed the portion size conditions did not moderate any of the effects in primary analyses (effect of portion size condition on intake from the served portion, additional energy intake, or total energy intake) in either Study 1 or Study 2. In Study 2, controlling for portion size order resulted in a minor deviation from the pattern of results in the primary analysis of additional intake such that the difference between the 'smaller than normal' and 'small-normal' portion size conditions was no longer significant, $m$ difference $=135 \cdot 0 \mathrm{~kJ}, \quad \mathrm{SE}=80 \cdot 7$, $P=0 \cdot 11$. See online Supplementary materials for full results.

\section{General discussion}

Across two experimental laboratory studies, in comparison with when served a 'small-normal' portion of food, we found evidence of greater additional eating when participants were served an initial portion that was visually perceived by an independent sample of participants as being 'smaller than normal'. This absolute effect size was small but statistically significant in the main analyses of Study 1 and in sensitivity analyses in Study 2 . We found less evidence of a difference in additional intake associated with the same-sized difference in portion size between a 'large-normal' portion and one that was smaller but still perceived as 'normal'. However, despite evidence of greater additional intake observed after consuming a 'smaller than normal' than a 'small-normal' portion, participants did not fully compensate for the difference in energy consumed from the initial portion: total meal energy intake including ad libitum intake of additional food was still significantly lower after consuming a 'smaller than normal' portion. Furthermore, despite smaller portions being associated with consuming significantly less energy than larger portions, there were no accompanying differences in participants' self-reported post-meal hunger or fullness between portion size conditions, in line with some previous findings ${ }^{(14,28-30)}$ but not others ${ }^{(10,16,31)}$.

There were some differences between the two studies. Participants in Study 2 consumed more energy than participants in Study 1. There are several factors that may have contributed to this behaviour. First, the two-course meal structure in Study 2 may have conveyed to participants that consuming at least some dessert was normative or expected. Second, due to the wider variety of food available to participants, sensory-specific satiation is less likely to have constrained additional intake. These factors may have dampened the influence of perceived normality of the portion size of the served meal component in Study 2 relative to Study 1 and may explain why our predictions about portion size normality were more clearly supported in Study 1 than Study 2. Other differences (e.g. sensory experience, macronutrient composition) between the meals provided in the studies could have also contributed to differences in the pattern of results.

There are some caveats to interpreting the results of the present studies in relation to the 'norm range' model. In line with the norm range model, perceptions of portion size normality tended to be negatively correlated with the amount of additional intake in unplanned analyses. However, participants' end-ofstudy ratings of the 'large-normal' portion sizes (and the 'small-normal' portion in Study 2) were significantly higher than 'normal', and participants' self-reported 'usual' portion size of the study foods were closer to the 'smaller than normal' than the 'normal' portion sizes in Study 2. These findings indicate that the served portion sizes may have been too large for some participants; however, this may also be attributable to measurement issues. The portions were selected based on visual judgments of portion size 'normality' from an independent sample of participants in a previous study ${ }^{(23)}$. We adopted this approach to prevent hypothesis awareness that may have arisen from participants in the present studies assessing perceived normality at the start of the study, which was successful as only a small number of participants were aware of the true hypothesis of 
the studies. We believe this approach was justified as we have previously observed considerable overlap between the perceived 'norm range' of independent samples and we employed the same recruitment strategy and stratification ${ }^{(23)}$. Prior exposure to and consumption of different portion sizes has now been shown to affect perceived normality ${ }^{(18,32,33)}$, meaning these measures completed at the end of the studies may have been contaminated by exposure to portion sizes during the study. Further, portion size normality ratings were assessed consecutively, which may have artificially inflated the differences between the portion size conditions (e.g. participants may have evenly spaced the ratings along the scale, rather than clustering in the middle of the scale around perceived 'normal' as was predicted). An alternative interpretation is that despite these methodological difficulties, perceived normality does not have a significant influence on additional intake, and as such, future research corroborating the preliminary evidence for a 'norm range' interpretation of additional intake following the consumption of a moderate to small initial portion of food would be valuable. This could be achieved using a between-subjects design to simultaneously minimise hypothesis awareness and allow cleaner measurement of perceived portion size normality, or by manipulating perceived portion size normality to circumvent the issue of measurement contamination.

The present work adds to the evidence that reducing portion size can reduce short-term energy intake and provides tentative preliminary empirical support regarding one factor that may influence the boundaries of effective portion size reductions. However, we note that further research addressing the methodological limitations acknowledged above is required to provide more convincing evidence. Some previous work has shown that reducing portion size decreases acute energy intake, and this decrease is not fully compensated for by consumers with increased intake from side dishes ${ }^{(34)}$ or at later meals ${ }^{(14,15)}$. However, in a 6-month free-living randomised controlled trial, a reduction of weekday lunch portions from 800 to $400 \mathrm{kcal}$ (3347 to $1674 \mathrm{~kJ}$ ) was associated with neither significantly lower daily energy intake nor greater weight $\operatorname{loss}^{(15)}$, suggesting that if portion size is decreased by too much, compensation may occur. Our findings provide some preliminary support for a 'norm range' theoretical model of the effect of portion size ${ }^{(23)}$. Specifically, the model and our findings tentatively suggest that if a reduction in size results in a portion size being categorically perceived as a 'normal' sized portion, immediate additional eating may be less likely than if the portion size is perceived as 'smaller than normal'. Moreover, this model suggests that the exact size of reduction to any given food that could be made without inviting substantial additional eating may be reliant on the range of portion sizes of that food that most consumers visually perceived as being 'normal' in size, as opposed to a simple rule of thumb applied across all foods (e.g. a $50 \%$ reduction in portion size). It is important to note that our studies focused on additional eating after an initial portion both during the same course (Study 1) and in a second course of the same meal (Study 2), so our conclusions only reflect short-term patterns of intake and only part of overall energy balance. Further work is needed to examine longer-term patterns of additional intake in response to reduced portion sizes (e.g. over several days, as has been examined in children with standard $v$. 'increased' portions ${ }^{(35)}$ ), although a recent systematic review concluded that acute effects of dietary interventions on appetite and energy intake tend to be sustained if the initial effects are robust ${ }^{(36)}$. The relevance of the present work in explaining the effect that increases in portion size have on energy intake may now be valuable to examine.

A strength of the studies reported here is that detailed cover stories were used, which were successful at disguising the study aims from the majority of participants ${ }^{(37)}$. Both studies were wellpowered, and Study 2 was pre-registered and demonstrated generalisability of the pattern of results of Study 1 to a different food and different meal structure. We also provided participants with portion sizes that would be more representative of meals served outside of the laboratory, which increased ecological validity. For example, although plate clearing is very common when eating $^{(38)}$, for methodological reasons, traditional laboratory portion size studies are designed to be so large that participants are unable to finish them. We allowed a variable washout period (7-10 d) in order to facilitate scheduling and retention of participants, but a 7-d washout (sessions on same day of the week) would have been preferable. It is possible that outside of the laboratory setting, consumers may more freely engage in additional eating of self-chosen foods or outside of the single meal occasion (as evidenced in previous works, e.g. de Castro, McKiernan et al. and Champagne et al. ${ }^{(39-41)}$ ), which may result in different patterns of additional intake than was observed in the present research. There is now a need to test whether the pattern of results observed here can be reproduced in more naturalistic settings and by assessing patterns of food intake over longer time periods ${ }^{(42)}$. Another question for future research is the extent to which individual differences may affect additional intake after consumption of smaller portions, as the present studies were not designed to examine this. For example, satiety responsiveness ${ }^{(43)}$ may dictate the amount of additional intake, although the effect that portion size has on energy intake has not been shown to be consistently moderated by satiety responsiveness ${ }^{(35,44)}$. Although a strength of the present research was that we compared three different-sized portions inside and outside of the 'norm range' for two different foods and two different meal structures, comparing patterns of additional eating in response to a greater number of portion sizes varying in perceived normality will now be needed to provide a more robust test of the norm range model, and would be another useful direction for future research.

There are calls for policy action to encourage food manufacturers and retailers to reduce food portion sizes in order to reduce energy intake and tackle obesity ${ }^{(2,12)}$. However, it has been argued that variations in portion size are more likely to lead to compensatory responses than interventions relating to other aspects of eating behaviour such as ingestive frequency, meaning that portion size may be a less important consideration for overall energy intake and population weight gain ${ }^{(7,45)}$. Here we made subtle reductions to moderately sized portions and, 
despite evidence of a small increase in additional eating in response to portions perceived as being 'smaller than normal', participants did not fully compensate for the portion size reductions, suggesting that further reductions to portions that are already small-to-moderate in size have the potential to reduce overall energy intake.

\section{Conclusions}

Two studies provide preliminary evidence that perceived portion size normality may influence additional intake, but further research testing the effects on energy intake of manipulating a wider range of portion sizes that vary according to perceived normality over a longer period of time is required.

\section{Acknowledgements}

We are grateful to Rebecca Bianchi and Anne-Laure de Fornel for assistance with data collection.

This research was supported by a New Investigator Research Grant (NIRG) awarded by the Medical Research Council (MRC) to E. R. (MR/N00218/1). The funding body had no role in the design of the study or collection, analysis or interpretation of data or in writing the manuscript.

All authors contributed to designing the research: E. R. and A. H. oversaw data collection and analysed data; A. H. drafted the manuscript; and all authors contributed to the final written manuscript. All authors were responsible for the final approval of the manuscript.

E. R.'s salary is supported by the MRC; E. R. and J. C. G. H. have also received research funding from the American Beverage Association and Unilever; C. A. H. has received research funding from the American Beverage Association and speaker's fees from the International Sweeteners Association; S. A. J. is a National Institute for Health Research (NIHR) Senior Investigator and funded by the NIHR Oxford Biomedical Research Centre (BRC) and NIHR Collaboration for Leadership in Applied Health Research and Care Oxford at Oxford Health NHS Foundation Trust.

\section{Ethics approval and consent to participate}

This research received ethical approval from the University of Liverpool Institute of Psychology, Health and Society Research Ethics Committee (approval codes IPHS-1516-LB-252-Generic RETH000955, IPHS-1617-LB-277-Generic RETH000955) and all participants provided informed consent.

\section{Availability of data and materials}

The datasets supporting the conclusions of this article are available in the Open Science Framework repository (https://osf.io/txf9u/).

\section{Supplementary material}

Online supplementary materials contain a CONSORT flow diagram, additional methodological details, and additional details on secondary analyses. For supplementary material referred to in this article, please visit https://doi.org/10.1017/S000711 4519002307

\section{References}

1. Nielsen SJ \& Popkin BM (2003) Patterns and trends in food portion sizes, 1977-1998. JAMA 289, 450-453.

2. Marteau TM, Hollands GJ, Shemilt I, et al. (2015) Downsizing: policy options to reduce portion sizes to help tackle obesity. BMJ 351, h5863.

3. Young LR \& Nestle M (2002) The contribution of expanding portion sizes to the US obesity epidemic. Am J Public Health 92, 246-249.

4. Livingstone MBE \& Pourshahidi LK (2014) Portion size and obesity. Adv Nutr 5, 829-834.

5. Kelly MT, Rennie KL, Wallace JMW, et al. (2008) Associations between the portion sizes of food groups consumed and measures of adiposity in the British National Diet and Nutrition Survey. Br J Nutr 101, 1413-1420.

6. Pereira J, Félix P, Mattei J, et al. (2018) Differences over 12 years in food portion size and association with excess body weight in the city of São Paulo, Brazil. Nutrients 10, E696.

7. Herman CP, Polivy J, Vartanian LR, et al. (2016) Are large portions responsible for the obesity epidemic? Physiol Behav 156, 177-181.

8. Hollands GJ, Shemilt I, Marteau TM, et al. (2015) Portion, package or tableware size for changing selection and consumption of food, alcohol and tobacco. Cochrane Database Syst Rev, issue 9, CD011045.

9. Zlatevska N, Dubelaar C \& Holden SS (2014) Sizing up the effect of portion size on consumption: a meta-analytic review. J Marketing 78, 140-154.

10. Rolls BJ, Roe LS \& Meengs JS (2007) The effect of large portion sizes on energy intake is sustained for 11 days. Obesity $\mathbf{1 5}$, $1535-1543$

11. Jeffery RW, Rydell S, Dunn CL, et al. (2007) Effects of portion size on chronic energy intake. Int J Behav Nutr Phys Act 4, 27.

12. Steenhuis I \& Poelman M (2017) Portion size: latest developments and interventions. Curr Obes Rep 6, 10-17.

13. Steenhuis IH \& Vermeer WM (2009) Portion size: review and framework for interventions. Int J Behav Nutr Phys Act 6, 58.

14. Rolls BJ, Roe LS \& Meengs JS (2006) Reductions in portion size and energy density of foods are additive and lead to sustained decreases in energy intake. Am J Clin Nutr 83, 11-17.

15. French SA, Mitchell NR, Wolfson J, et al. (2014) Portion size effects on weight gain in a free living setting. Obesity 22, 1400-1405.

16. Lewis HB, Ahern AL, Solis-Trapala I, et al. (2015) Effect of reducing portion size at a compulsory meal on later energy intake, gut hormones, and appetite in overweight adults. Obesity 23, 1362-1370.

17. Kerameas K, Vartanian LR, Herman CP, et al. (2015) The effect of portion size and unit size on food intake: unit bias or segmentation effect? Health Psychol 34, 670.

18. Robinson E \& Kersbergen I (2018) Portion size and later food intake: evidence on the 'normalizing' effect of reducing food portion sizes. Am J Clin Nutr 107, 640-646.

19. Herman CP, Polivy J, Pliner P, et al. (2015) Mechanisms underlying the portion-size effect. Physiol Behav 144, 129-136.

20. Herman CP \& Polivy J (2005) Normative influences on food intake. Physiol Behav 86, 762-772.

21. Vartanian LR, Reily NM, Spanos S, et al. (2017) Hunger, taste, and normative cues in predictions about food intake. Appetite 116, 511-517.

22. Versluis I \& Papies EK (2016) The role of social norms in the portion size effect: reducing normative relevance reduces the effect of portion size on consumption decisions. Front Psychol 7, 756.

23. Haynes A, Hardman CA, Makin ADJ, et al. (2019) Visual perceptions of portion size normality and intended food consumption: a norm range model. Food Qual Prefer 72, 77-85. 
24. Rolls BJ, Rolls ET, Rowe EA, et al. (1981) Sensory specific satiety in man. Physiol Behav 27, 137-142.

25. NatCen Social Research, University College London, Department of Epidemiology and Public Health (2016) Health Survey for England, 2014 (data collection), 2nd ed. UK Data Service. https://beta.ukdataservice.ac.uk/datacata logue/doi?id=7919

26. Sawilowsky SS (2009) New effect size rules of thumb. J Mod Appl Stat Methods 8, 597-599.

27. Bakdash JZ \& Marusich LR (2017) Repeated measures correlation. Front Psychol 8, 456.

28. Kral TV, Roe LS \& Rolls BJ (2004) Combined effects of energy density and portion size on energy intake in women. Am J Clin Nutr 79, 962-968.

29. Roe LS, Kling SMR \& Rolls BJ (2016) What is eaten when all of the foods at a meal are served in large portions? Appetite 99, 1-9.

30. Zuraikat FM, Roe LS, Privitera GJ, et al. (2016) Increasing the size of portion options affects intake but not portion selection at a meal. Appetite 98, 95-100.

31. Diliberti N, Bordi PL, Conklin MT, et al. (2004) Increased portion size leads to increased energy intake in a restaurant meal. Obes Res 12, 562-568.

32. Robinson E, Oldham M, Cuckson I, et al. (2016) Visual exposure to large and small portion sizes and perceptions of portion size normality: three experimental studies. Appetite 98, 28-34.

33. Robinson E, Henderson J, Keenan GS, et al. (2019) When a portion becomes a norm: exposure to a smaller vs. larger portion of food affects later food intake. Food Qual Prefer 75, 113-117.

34. Carstairs S, Caton S, Blundell-Birtill P, et al. (2018) Can reduced intake associated with downsizing a high energy dense meal item be offset by increased vegetable variety in 3-5-year-old children? Nutrients 10, 1879.
35. Smethers AD, Roe LS, Sanchez CE, et al. (2019) Portion size has sustained effects over 5 days in preschool children: a randomized trial. Am J Clin Nutr 109, 1361-1372.

36. Halford JCG, Masic U, Marsaux CFM, et al. (2018) Systematic review of the evidence for sustained efficacy of dietary interventions for reducing appetite or energy intake. Obes Rev $\mathbf{1 9}$, 1329-1339.

37. Robinson E, Bevelander KE, Field M, et al. (2018) Methodological and reporting quality in laboratory studies of human eating behavior. Appetite 125, 486-491.

38. Robinson E \& Hardman CA (2015) Empty plates and larger waists: a cross-sectional study of factors associated with plate clearing habits and body weight. Eur J Clin Nutr 70, 750.

39. de Castro JM (1998) Prior day's intake has macronutrientspecific delayed negative feedback effects on the spontaneous food intake of free-living humans. J Nutr 128, 61-67.

40. McKiernan F, Hollis JH \& Mattes RD (2008) Short-term dietary compensation in free-living adults. Physiol Behav 93, 975-983.

41. Champagne CM, Han H, Bajpeyi S, et al. (2013) Day-to-day variation in food intake and energy expenditure in healthy women: the dietitian II study. J Acad Nutr Diet 113, 1532-1538.

42. Best M, Barsalou LW \& Papies EK (2018) Studying human eating behaviour in the laboratory: theoretical considerations and practical suggestions. Appetite 130, 339-343.

43. Zuraikat FM, Roe LS, Smethers AD, et al. (2018) Does the cost of a meal influence the portion size effect? Appetite 127, 341-348.

44. Zuraikat FM, Roe LS, Smethers AD, et al. (2018) Doggy bags and downsizing: packaging uneaten food to go after a meal attenuates the portion size effect in women. Appetite 129, 162-170.

45. Mattes R (2014) Energy intake and obesity: ingestive frequency outweighs portion size. Physiol Behav 134, 110-118. 